\title{
Students' Perspectives Following Involvement in a Constraint Induced Aphasia Therapy Research Project
}

Bridget R. Dincher

Ball State University, bdincher@bsu.edu

Melissa McGrath

Ball State University, mamcgrath@bsu.edu

Julie Griffith

Ball State University, jgriffith2@bsu.edu

DOI: https://doi.org/10.30707/TLCSD4.2/SMWG7415

Follow this and additional works at: https://ir.library.illinoisstate.edu/tlcsd

Part of the Other Rehabilitation and Therapy Commons, and the Scholarship of Teaching and Learning Commons

\section{Recommended Citation}

Dincher, Bridget R.; McGrath, Melissa; and Griffith, Julie (2020) "Students' Perspectives Following Involvement in a Constraint Induced Aphasia Therapy Research Project," Teaching and Learning in Communication Sciences \& Disorders: Vol. 4: Iss. 2, Article 2.

DOI: https://doi.org/10.30707/TLCSD4.2/SMWG7415

Available at: https://ir.library.illinoisstate.edu/tlcsd/vol4/iss2/2

This Student Voices is brought to you for free and open access by ISU ReD: Research and eData. It has been accepted for inclusion in Teaching and Learning in Communication Sciences \& Disorders by an authorized editor of ISU ReD: Research and eData. For more information, please contact ISUReD@ilstu.edu. 


\title{
Students' Perspectives Following Involvement in a Constraint Induced Aphasia Therapy Research Project
}

\author{
Abstract \\ The purpose of this project was to provide a reflection on four students' collaborative research experience \\ implementing Constraint-Induced Aphasia Therapy and highlight the importance of student involvement \\ in research opportunities. Guided reflections were completed and analyzed through a collaborative model \\ to generate common themes of: 1) increased confidence in cuing and 2) adapting individualized \\ treatment. Student engagement in clinical research enhances the quality of their educational experience \\ by fostering clinical competence and confidence.
}

\section{Keywords}

Student perspectives, student research opportunities, collaboration

\section{Cover Page Footnote}

We would like to thank Laura Norris, Caroline Schoeff, and Whitney Wooten who were contributors on this project. 
Clinical research opportunities provided by graduate programs in communication sciences and disorders create a platform for collaborative problem-based learning (Ginsberg et al., 2012). Collaboration between teachers and students is a fundamental goal of the scholarship of teaching and learning (SOTL) (Allin, 2014), which traditionally explores pedagogical methods to improve student education. SOTL research excels in evaluating the efficacy of teaching interventions. A challenge of clinical education is preparing students for practice by bridging theory discussed in the classroom to their therapeutic practice (Dalton et al., 2017). Collaboration with students is necessary to gauge their understanding of theory for effective use of therapeutic strategies. Students' perspectives are important in influencing larger programmatic designs within higher education to shape curriculum and best prepare students for clinical practice (Trowler \& Trowler, 2010). Previous work on clinical growth for speech-language pathology students has focused on topics such as reflective practice (Caty et al., 2015) and clinical decision making (Dudding \& Pfeiffer, 2018) from faculty perspectives. However, work regarding student growth from students' perspectives, in specific clinical skills such as cueing, goal writing, and documentation is limited in the communication sciences literature. The aim of the current project was to provide a student voice for clinical education in speech-language pathology through participation in a research study. The project is a reflection on four students' collaborative research experience implementing Constraint Induced Aphasia Therapy (CIAT) and highlights the importance of student involvement in research opportunities for future clinical practica.

\section{CIAT Research Study Description}

The graduate student clinicians participated in a research project by providing CIAT to eight clients who were individuals with chronic aphasia and placed in two groups of four under the supervision of two assistant professors. The larger research project took place over a six-week period during a fall semester. The students learned that CIAT was an intensive group therapy for individuals with chronic aphasia, which was founded on three principles: constraint, intensity or massed-practice, and shaping, to overcome learned non-use (e.g., Griffith et al., 2017; Meinzer et al., 2012; Szaflarski et al., 2015). The clients with aphasia were constrained by encouraging them to use the verbal modality and reduce reliance on gestures, finger spelling, and writing when communicating (Pulvermuller \& Berthier, 2008). The protocol included an average of 22 hours of therapy over five consecutive days in order to meet the second principal, intensity. Throughout the treatment the clients with aphasia played a language game much like 'go fish' during which they were required to verbally request picture cards from each other to create matches. The student clinicians utilized the shaping techniques to ensure successful communicative exchanges which included increasing the difficulty of linguistic output and decreasing the level of support provided (Griffith et al., 2017).

\section{Methodology}

A qualitative phenomenological research design (Creswell et al., 2007) was employed to guide the student clinicians' reflections of providing CIAT therapy during a clinical research project. Specifically, a guided reflection illuminated their common experiences and generated themes. The reflection was administered in written form and consisted of both open-ended and yes/no questions to obtain a variety of thoughts and opinions. (See appendix for the specific guided reflection questions.) 
Participants. The participants were four graduate student clinicians. They were all in their fourth semester of a graduate communication sciences and disorders program and had an average of three semesters of clinical experience in a university clinic. Out of the four student clinicians, only one had previously worked with a client with aphasia. The student clinicians had mostly treated children with speech sound disorders in a 1:1 setting for 50 to 90 minute sessions. The students chose to participate in the research opportunity because of a shared interest in working with people with aphasia and the rare opportunity it presented. In other words, at the time of this project, the university clinic had limited opportunities to provide group therapy and work with individuals with aphasia, and no mechanism which allowed student clinicians to treat clients simultaneously. Although it was the students' first experience with this type of therapy, they were in their last semester of university clinicals and considered experienced student clinicians.

An announcement regarding an opportunity to participate in a research project investigating CIAT was given to graduate students currently enrolled in an aphasia course. Interested students were instructed to email the primary investigator and the university clinic director to declare their desire to participate in the project. Students whose schedule allowed for an intensive treatment and needed adult clinical practicum hours were contacted to be a part of the project. Prior to the onset of the CIAT intervention, the student clinicians completed eight-hours of training. Training consisted of instruction on aphasia assessments, CIAT background, protocol implementation, video examples, and role-playing with a language cueing hierarchy. The cueing hierarchy transitioned from most to least amount of support, moving from repetition to reminder cues (Griffith et al., 2017). Each student clinician administered pre- and post-assessments and contributed equally during the CIAT intervention by working one-on-one with a client with aphasia. The students administered the CIAT protocol by providing shaping cues, limiting communication to the verbal modality and massed-practice to bolster the communicative success of the clients with aphasia. The clients with aphasia consisted of eight individuals between the ages of 55 - 80 years old who ranged in severity from mild anomic aphasia with mild dysarthria to severe Broca's aphasia with apraxia of speech. At the end of each CIAT treatment day, the student clinicians completed daily documentation, which included SOAP notes as well as the creation and/or modification of linguistic and social goals during a collaborative conference with all students and supervisors.

Guided Reflection. One week after the completion of the research protocol, the four graduate student clinicians independently completed a guided reflection on their experience as student clinicians. The guided reflection was created a priori by the principle investigator of the CIAT study, Dr. Julie Griffith, to gain valuable feedback on the CIAT program and guide the students' reflection of their learning. Each student clinician wrote responses to the questions posed by the guided reflection. The reflections were then collected and reviewed by the research team to generate common themes.

Analysis. The guided reflections were qualitatively analyzed in a three-step process by two graduate student clinicians, who were involved in the treatment. First, the students were blinded to who wrote each comment to diminish potential bias. However, since both individuals analyzing the reflections also completed a reflection of their own, potential bias remains and students may have reflected back on their own experience during the research project further while analyzing. 
Second, the reflections were read through independently to identify and record thematic ideas mentioned frequently by a majority of the student clinicians. After a period of time, the two students returned to their initial thematic impressions to collapse minor themes in order to develop larger themes and ensure stability of initial findings. Lastly, the two graduate students reviewed their findings together to determine the validity of the discovered themes. Through their collaboration, two common themes emerged and were presented to the two faculty supervisors to triangulate the students' learning.

\section{Results}

Theme: Increased Confidence in Cueing. The theme reported by the student clinicians with the highest frequency was cueing. "At the start, I was tending to only give phonemic cues, regardless of the client's individual needs, I was only giving cues that felt safe to me" (P4). The student clinicians gave many phonemic cues because they were the most straightforward and were most familiar. However, throughout the intervention, other types of cues became more familiar to them, (e.g., semantic phrase completion, choice of two, and reminders) and with increased familiarity, came confidence and use. The ability for the student clinicians to watching each other was the key factor in building their confidence. Their gained confidence allowed them to be more creative with the cues provided. As intervention went on, the student clinicians "paid more attention to each other's strategies and implemented what was working for them [the client's with aphasia]" (P1). After the student clinicians became confident in types of cueing, they started to think about the timing of them. The students realized that giving more cues is not always better, and sometimes "all they [the client's with aphasia] needed was encouragement" (P3). As the student clinicians started to recognize the importance of when cues are provided, they learned the hard lesson that it is "okay to wait and let the participant struggle" (P1). If a clinician continuously jumps in to help when things get a little difficult, the clients with aphasia do not challenge themselves or receive the practice required to reach their full potential. Along with decreasing the number of cues to foster greater independence, the student clinicians learned which type of cues were beneficial for each client with aphasia depending on their language level and type of aphasia.

Theme: Adaptation of Individualized Treatment - Goals and Documentation. The theme of adapting treatment first emerged in the student clinicians' reflections on cueing, specifically how each CIAT client required different frequency and types. Each client with aphasia was unique and, although they were completing the same tasks each day, they required individual goals due to differing linguistic levels of complexity based on their abilities. One student clinician described goal writing as "a balance between making [the goals] specific and general as to not put yourself in a box but allowing others to understand the direction of the goal" (P2). In other words, the student clinicians learned that goals should be flexible enough to allow for targeting in a variety of ways, yet specific enough that another clinician would easily understand how to provide therapy. However, writing goals to be specific is only one factor, "the most important considerations should always be what the client wants and needs and be functional" (P1). The student clinicians also learned that as participants make progress, goals need to be modified throughout treatment to keep them at an appropriate language level. Sometimes, clinicians modified goals during the day by collaborating and "building off of what our fellow clinicians did previously with a participant" (P3). After the end of each treatment day, the student clinicians documented the clients with aphasias' progress and held a collaborative conference to adjust goals 
as needed. One major aspect discussed during the end of the day collaborative meetings was the goals "adjusting [goals] for each participant and making [the goals] more difficult or easier depending on [the CIAT client's] progress" (P3). Discussion of the goals helped the student clinicians to write their reports more efficiently and document progress more clearly. One student clinician learned the importance of clear goals and documentation when they stated, "tracking data does not necessitate meticulous notation, but rather a representative sample” (P4), which they used to discuss the key aspects of each client's progress. During the students' previous clinic experiences, goals would be written within a week and often edited a few times before finalized. This project forced the student clinicians into a more real-world scenario where goals had to be written within a few minutes at the end of a treatment session before the next day. The nature of goal writing during this project facilitated an opportunity for increased competence compared to a typical university clinic practicum experience.

Faculty Supervisors' Reflection. The faculty members reflected on their own observations of the students' growth over the course of the larger project and responded to the students' identified reflective themes to provide further triangulation and insight.

Theme: Increased confidence in cueing. Prior to this research experience, the students had only engaged in traditional individualized treatment sessions in which they practiced using cueing strategies with a single client during an entire semester's clinical experience. Having the opportunity to provide cueing to multiple clients within the group setting enabled the students to refine their use of cueing strategies through both observation and practice. Clinical supervisors provided daily written feedback which logged type and frequency of cues in addition to students' daily SOAP notes. This clinical observation and documentation demonstrated that each student clinician increased in both type and frequency of cues utilized. They also provided cues with appropriate timing to maximize the clients' verbal output. The subtle nuances of useful cueing are difficult clinical skills to achieve. During this project, it was apparent that each student increased in clinical competence, which was evidenced by their ability to provide the most appropriate level of cueing to optimally challenge each client. This type of student progress is often difficult to achieve in more traditional clinical practicum settings.

Theme: Adaptation of individualized treatment - goals and documentation. The intensity of each day of treatment facilitated clinical growth for the student clinicians in their ability to write and adjust goals as well as to efficiently document progress. The student clinicians received valuable practice adjusting goals daily based upon each client's progress. Practice adjusting treatment goals to appropriately challenge clients is another clinical experience that graduate students in traditional practica, with less intensive treatment schedules, often lack. In addition, each student clinician learned firsthand the importance of both brevity and specificity in their daily documentation. Documenting at the end of a long day challenged the students' endurance but was an excellent opportunity to mimic note writing in a "real world" setting.

\section{Student Conclusion}

All of the student clinicians reported they would partake in a project similar to this again and encourage other universities to allow students opportunities to be a part of clinical research projects. This research project provided experience in an alternative setting which would not have 
been possible otherwise. Not only were the student clinicians able to log a substantial number of required adult/geriatric language hours, the study allowed for the opportunity to watch fellow clinicians work with the same clients. Observing one another and working with the same clients created a much different learning environment than one-on-one clinical sessions. Important factors contributing to the student clinicians' clinical growth were collaborating, especially with the same client, the high intensity of treatment, and the opportunity to treat a variety of people with aphasia in a short amount of time. All the student clinicians agreed that they had been adequately trained, however reported a lack of confidence in skills. The lack of confidence when starting the project may be attributed to the fact that application is different than theory. The theory, or being a student and knowing material for class is a necessary part of education; however, application, or applying that information within the clinical setting is a different situation entirely. Over the course of the research project, the students' confidence grew, they became more relaxed, and even started trusting their "clinical gut." After this project, the student clinicians were able to functionally apply the material learned in class to real-life clients with aphasia. Seeing aphasia and apraxia in real clients bridged the gap between being a student in the classroom and a student clinician. Through participation in this research project, the student clinicians are now confident in their ability to treat clients with aphasia, write individualized goals and provide the most beneficial cues. Any time that a faculty member has a clinical research project, the university should encourage student involvement in hopes to expose them to collaborative situations, which further improves students' education. Engaging in clinical research increased the quality of the students' educational experience and fostered greater confidence in them as future speech-language pathologists.

\section{Faculty Conclusion}

Taking on dual roles as researchers and clinical supervisors, the primary investigators sought to complete a study utilizing CIAT to enhance the communication skills of people with aphasia. Including four graduate students in the research allowed the protocol to provide 1:1 clinical support for each client with aphasia. A beneficial byproduct of the larger research study was the observed clinical growth in each of the graduate student clinicians. Throughout the larger project, the students experienced the dynamics of providing group therapy in a collaborative and intensive manner. Since investigations on clinical growth for speech-language pathology students has mostly provided faculty perspectives (Caty et al., 2015; Dudding \& Pfeiffer, 2018), this refection reveals how the students perceived the development of their clinical skills. The clinical growth discussed in this paper from the students' reflective experience highlights how group treatment and collaboration foster the clinical skills of cueing, goal writing, and especially confidence. Even more, the students' clinical growth was substantiated by supervisor feedback and observation. The skills the graduate students gained from their involvement in a clinical research project are unique and difficult to replicate in the classroom or more traditional individual clinic sessions. Previous work regarding student involvement in research has centered around end goals of increased student interest in research careers (Ramjiawan et al., 2012) or increasing the appreciation of the differences between research in practice and research in text (Pietersen, 2002). Our findings indicated that student participation in the implementation of therapeutic research protocols also improved their clinical skills. Future inclusion of students in the research process is highly encouraged. The collaboration gives students experiences beyond the traditional clinical setting and provides not only invaluable research understanding, but opportunities for clinical growth. 


\section{Financial disclosure statements}

Bridget Dincher was employed by Ball State University, at which this project took place until December 2019. She was also student at Ball State University until July 2019.

Melissa McGrath is employed by Ball State University, at which this project took place. This is a continuing relationship.

Julie Griffith is employed by Ball State University, at which this project took place. This is a continuing relationship until May 2020.

\section{Nonfinancial disclosure statements}

Bridget Dincher has no nonfinancial disclosures.

Melissa McGrath has no nonfinancial disclosures.

Julie Griffith has no nonfinancial disclosures.

\section{References}

Allin, L. (2014). Collaboration between staff and students in the Scholarship of Teaching and Learning: The potential and the problems. Teaching \& Learning Inquiry: The ISSOTL Journal, 2(1), 95 - 102.

Creswell, J. W., Hanson, W. E., Clark Plano, V. L., \& Morales, A. (2007). Qualitative Research Designs: Selection and Implementation. The Counseling Psychologist, 35(2), 236-264.

Caty, M. E., Kinsella, E. A., \& Doyle, P. C. (2015). Reflective practice in speech-language pathology: A scoping review. International Journal of Speech-language Pathology, 17(4), 411-420.

Dalton, J. C., Klein, J., \& Botts, D. C. (2017). Bridging the divide with SoTL: Connecting knowledge with best practice in communication disorders. Perspectives of the ASHA Special Interest Groups, 2(10), 14-24.

Dudding, C. \& Pfeiffer, D. (2018) Clinical decision-making in speech-language pathology graduate students: Quantitative Findings. Teaching and Learning in Communication Sciences \& Disorders, 2(1), Article 2. https://ir.library.illinoisstate.edu/tlcsd/vol2/iss1/

Ginsberg, S. M., Friberg, J. C. \& Visconti, C. F. (2012). Scholarship of Teaching and Learning in Speech-Language Pathology and Audiology: Evidence Based Education. San Diego, CA: Plural Publishing Inc.

Griffith, J., Dietz, A., Ball, A., Vannest, J., \& Szaflarski, J. P. (2017). An examination of changes in spoken productions within constraint-induced aphasia therapy. Aphasiology, $31(11), 1250-1265$.

Meinzer, M., Rodriguez, A. D., \& Gonzalez Rothi, L. J., (2012). First decade of research on constrained-induced treatment approaches for aphasia rehabilitation. Archives of Physical Medicine and Rehabilitation, 93(1), S35-S45. doi:10.1016/j.apmr.2011.06.040. 
Pietersen, C. (2002). Research as a learning experience: A phenomenological explication. The qualitative report, 7(2), 1-14.

Pulvermüller, F., \& Berthier, M. L. (2008). Aphasia therapy on a neuroscience basis. Aphasiology, 22(6), 563-599. doi:10.1080/02687030701612213

Ramjiawan, B., Pierce, G. N., Anindo, M. I. K., AlKukhun, A., Alshammari, A., Chamsi, A. T., ... \& Ganguly, P. K. (2012). An international basic science and clinical research summer program for medical students. Advances in physiology education, 36(1), 27-33.

Szaflarski, J., Ball, A., Vannest, J., Dietz, A., Allendorfer, J., Martin, A., ... Lindsell, C. (2015). Constraint-Induced Aphasia Therapy for Treatment of Chronic Post-Stroke Aphasia: A Randomized, Blinded, Controlled Pilot Trial. Medical Science Monitor: International Medical Journal for Experimental and Clinical Research., 21, 2861-2869. https://doi.org/10.12659/MSM.894291

Trowler, V., \& Trowler, P. (2010). Student engagement evidence summary. The Higher Education Academy, 1-30. 


\section{Appendix}

Student Clinician Guided Reflection

Directions: Please answer the following questions by reflecting back on your time as a CIAT clinician.

1. Do you feel as though the participants' communicative abilities changed over the course of the program? Please explain.

2. What, in your opinion, contributed most to any communicative changes that you may have observed?

3. Would you implement CIAT in the future? YES or NO

4. Please explain your response to 3, if yes, by stating what you would do the same or different.

5. What motivated you to be a part of this project?

6. How did you grow as a clinician from the pre- to post-assessment sessions?

7. How did you grow as a clinician from week 1 to week 2 of treatment?

8. How did you grow as a clinician in the type and amount of cues given?

9. What have you learned about writing and adjusting goals? 